\title{
An Improved Phase Method for Time-Delay Estimation
}

Svante Björklund, Lennart Ljung

Division of Automatic Control

E-mail: svabj@foi.se, ljung@isy.liu.se

20th December 2010

Report no.: LiTH-ISY-R-2985

Accepted for publication in Automatica, Vol 45, pp 2467-2470, 2009.

Address:

Department of Electrical Engineering

Linköpings universitet

SE-581 83 Linköping, Sweden

WWW: http://wwW.control.isy.liu.se

AUTOMATIC CONTROL

REGLERTEKNIK

LINKÖPINGS UNIVERSITET

Technical reports from the Automatic Control group in Linköping are available from http://www . control.isy.liu.se/publications. 


\begin{abstract}
A promising method for estimation of the time-delay in continuous-time linear dynamical systems uses the phase of the allpass part of a discrete-time model of the system. We have discovered that this method can sometimes fail totally and we suggest a method for avoiding such failures.
\end{abstract}

Keywords: System Identification 


\title{
An Improved Phase Method for Time-Delay Estimation *
}

\author{
Svante Björklund ${ }^{\mathrm{a}}$, Lennart Ljung ${ }^{\mathrm{b}}$ \\ ${ }^{a}$ Swedish Defence Research Agency, Box 1165, SE - 581 11, Linköping, Sweden \\ ${ }^{\mathrm{b}}$ Division of Automatic Control, Department of Electrical Engineering, Linköping University, SE-581 83 Linköping, Sweden
}

\begin{abstract}
A promising method for estimation of the time-delay in continuous-time linear dynamical systems uses the phase of the allpass part of a discrete-time model of the system. We have discovered that this method can sometimes fail totally and we suggest a method for avoiding such failures.
\end{abstract}

Key words: time-delay; dead-time; Laguerre; allpass; phase; zero; system identification; estimation

\section{Introduction}

Estimation of time-delays (or dead-times) in linear dynamical systems with additive noise is a common engineering problem, e.g. in control performance monitoring of industrial processes, in design and tuning of controllers, in range estimation in radar and in direction finding in signal intelligence. It is also a necessary part in all system identification.

In Horch (2000); Isaksson et al. (2001) a time-delay estimation method is described: A discrete-time model of a continuous-time system is identified using a Laguerre basis. The discrete-time non-minimum phase zeros of the model constitute the allpass part of the model and directly represent the time-delay. The time-delay is estimated by studying the slope at low frequencies of the phase of the allpass part. The method shows very good results in both open loop and closed loop for input and reference signals in the form of steps in Horch (2000); Isaksson et al. (2001). This method is in this paper called Laguerre DAP. The part of the method from the discretetime model to the time-delay estimate is here called the $D A P$ (Discrete-time Allpass part Phase) method.

In this paper we show that the DAP method is nonrobust and we improve the method to also handle some cases where it otherwise totally fails. A more thorough

\footnotetext{
* This paper was not presented at any IFAC meeting. Corresponding author Svante Björklund. Tel. +46-13-378000, Fax. +46-13-378100.

Email addresses: svabj@foi.se (Svante Björklund), ljung@isy.liu.se (Lennart Ljung).
}

description of the work underlying this paper is given in Björklund (2002).

\section{The DAP method}

\subsection{The principles of the DAP method}

Assume the true continuous-time system is $\bar{G}(s)=$ $\bar{G}_{1}(s) \cdot e^{-s T_{d}}=\bar{G}_{1}(s) \cdot \bar{G}_{a p}(s)$. The system $\bar{G}_{1}(s)$ is a SISO (single-input single-output) time-invariant linear rational transfer function. A discrete-time rational linear model $G(z)$ of $\bar{G}(s)$ is estimated and factorized into a minimum-phase system $G_{1}(z)$ and an allpass system $G_{a p}(z)$ as $G(z)=G_{1}(z) G_{a p}(z)$. Then, $G_{a p}\left(e^{i \omega T_{s}}\right)$ is considered to be an approximation of the time-delay system $\bar{G}_{a p}(i \omega)=e^{-i \omega T_{d}}$ (Horch, 2000; Isaksson et al., 2001), which should agree well for low frequencies since the frequency function of a sampled system agrees well with its continuous counterpart for low frequencies (Ljung and Glad, 1994). $T_{s}$ is the sampling interval. The allpass part $G_{\mathrm{ap}}(z)$ of $G(z)$ is formed by the nonminimum phase (positioned outside the unit circle) zeros of $G(z)$ and with poles added to $G_{\text {ap }}(z)$ which are these zeros mirrored in the unit circle.

The time-delay estimate $\hat{T}_{d}$ (in number of sampling intervals) is given by an approximation of the derivative of the phase $\varphi(\omega)=\arg G_{a p}\left(e^{i \omega T_{s}}\right)$ at the frequency zero and with addition of 1 because of the extra time delay that is created by the sampling:

$$
\hat{T}_{d}=-\frac{\arg G_{a p}\left(e^{i \omega_{1} T_{s}}\right)}{\omega_{1} T_{s}}+1 ; \quad \omega_{1} \ll 1
$$


This is the DAP method. Of course, (1) cannot be evaluated for $\omega_{1}=0$. In Horch (2000); Isaksson et al. (2001) $\omega_{1}=10^{-4}$ is suggested since it "has been found to be sufficiently small when using typical industrial data". This value is also used in this article.

Other approximations of the derivative should also be possible. In Horch (2000); Isaksson et al. (2001) a Laguerre model (Wahlberg, 1991) was used. Other linear model structures, for example FIR, ARX or OE (output error) model structures (Ljung, 1999) can also be used.

\subsection{A problem with the DAP method}

First, an example of when the DAP method works well is given. The linear continuous-time system $G_{2}(s)=$ $e^{-9 s} \cdot \bar{G}_{2}(s)$, where $\bar{G}_{2}$ had poles $-1 \&-10$ and no zeros (a fast second order system), was simulated in MATLAB by the function Isim CSTB (4.2.1) with an input consisting of a narrowband (most energy between $10 \%$ and $30 \%$ of the Nyquist frequency) random binary signal of length 500 samples. This input signal type is common in system identification if the signal can be chosen freely. To the output signal, white Gaussian noise was added and the signal-to-noise ratio (SNR) was 10 . The SNR was defined as the ratio of the powers of the input signal and of the output noise. The sampling interval was $T_{s}=1$ time units. A discrete-time Laguerre model with pole $\alpha=0.8$ and $N_{l}=10$ coefficients was identified from the input-output data and the DAP method was used to estimate the time-delay. The simulation resulted in the poles and zeros of the identified Laguerre model depicted in Figure 1. The time-delay estimate became 9.7114 sampling intervals, which is a good estimate since the true time-delay is 10 .

Now we turn to a case when the time-delay estimation failed. The only difference to the successful trial in the setup was a different noise realization. The zeros and poles of the identified Laguerre model are shown in Figure 2. We note that the zero on the real axis just inside the unit circle in the successful simulation has moved to just outside the unit circle. The time-delay estimate became 1321.86 sampling intervals, which is completely wrong. If we remove the moved zero, the time-delay estimate will be 11.6586 , which is an acceptable estimate.

The simulation in Figure 2 was one of only 3 out of 1024 simulations with failing time-delay estimation for SNR $=10$. With a lower SNR the percentage of failing estimations is much higher. Compare with Figure 3. In Björklund $(2002,2003)$ simulations indicated that the failure rate depends on the model structure (e.g. Laguerre, OE, ARX and FIR), the input signal type (e.g. steps or random binary signals with different bandwidths) and the SNR. For example, Laguerre models with step input signals gave a low failure rate while narrowband random binary input signals gave a high.

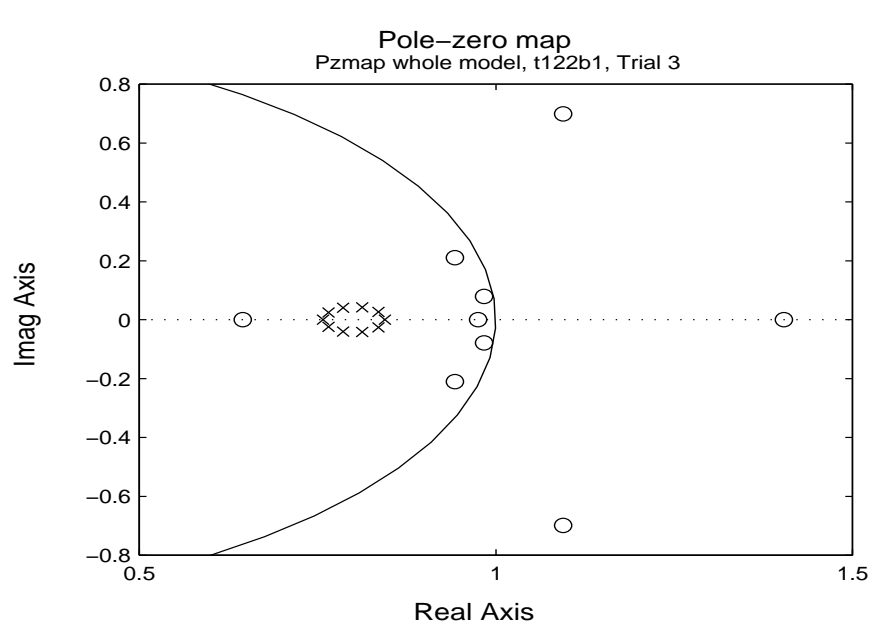

(a) Whole model

Figure 1. Poles (x) and zeros (o) plot of an identified Laguerre model (10 coefficients, pole at 0.8 ) for a subsequent successful time-delay estimation ( $\hat{T}_{d}=9.7114$ sampling intervals). The Laguerre model was identified from the input-output data. No zero guarding was used (see section 2.3). The poles of the model should all be located at $\alpha=0.8$ but due to well-known numerical problems with multiple poles they are somewhat spread in the figure.

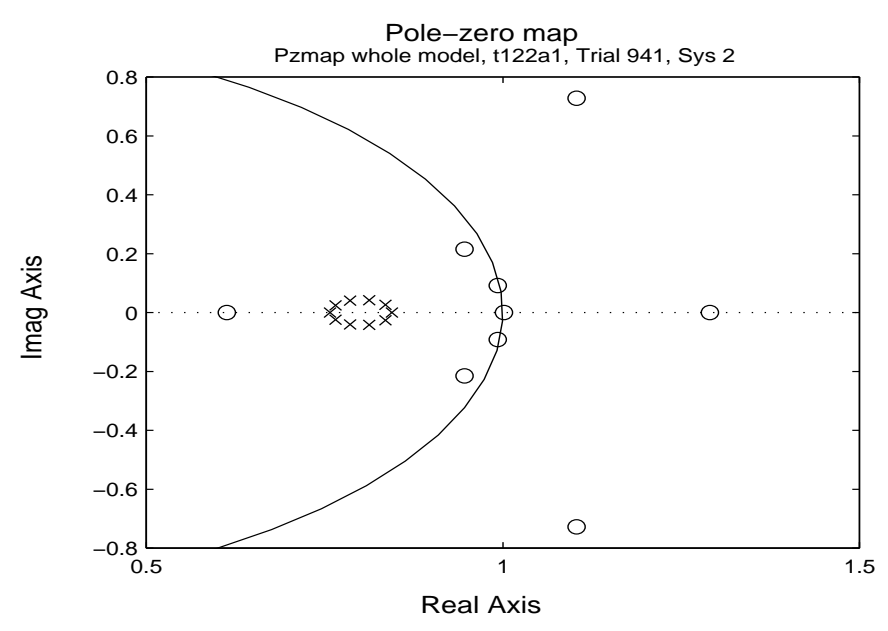

Figure 2. Pole-zero plot of an identified Laguerre model for a subsequent failing time-delay estimation $\left(\hat{T}_{d}=1321.86\right.$ sampling intervals). The simulation setup is the same as in Figure 1. Only the noise realization differs.

\subsection{A solution for the DAP method}

The reason for zeros falling on the incorrect side of the unit circle is the noise. Figure 3 shows an example of the spread of zeros and poles due to the noise. Figure 4 displays the time-delay estimate for different locations of the zero closest to the point +1 . The maximum estimation error will occur for the smallest error in the position of a zero that is incorrectly outside the unit circle. See Björklund $(2002,2003)$ for an explanation of how the estimated time-delay values are created. 


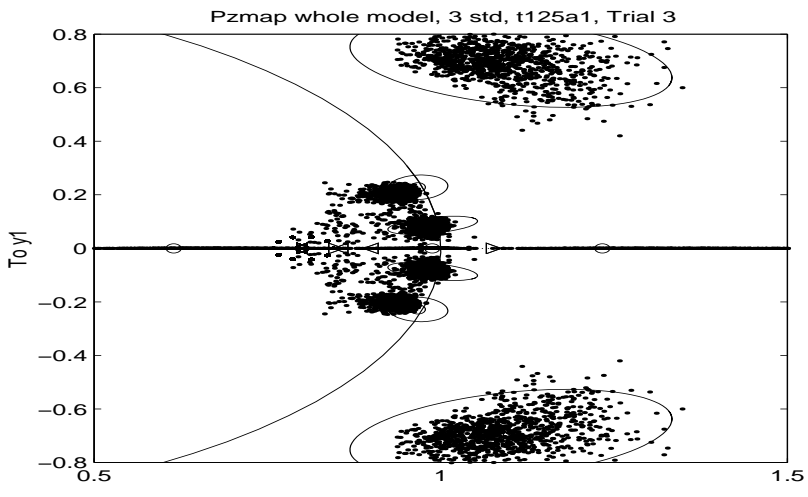

Figure 3. Pole-zero plot of a Laguerre model (10 coefficients, pole at 0.8 ) with estimated uncertainty regions (3 standard deviations) and with zeros and poles from 1024 simulated trials for $\mathrm{SNR}=1$. As can be seen, the risk of a zero falling on the wrong side of the unit circle is significant. The simulation setup is as in Figure 1 and 2.

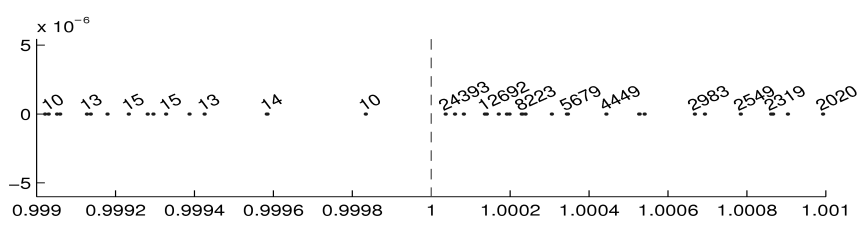

Figure 4. The graph covers an area close to the point +1 . The dots are locations of the zero closest to the point +1 for some simulations with different noise realizations. The numbers above the dots are the time-delay estimate corresponding to the zero location below it. It can be shown that the maximum possible estimate in this case is 31416 (Björklund, 2002, 2003). A Laguerre model was estimated. The true time-delay is 10 sampling intervals.

It appears that moving zeros located close to but outside the unit circle (back) to the inside of the unit circle is a solution to the problem with the DAP method. The motivation is that we assume that these zeros actually should be located inside the unit circle. Since we only need the allpass part in the DAP methods, we just remove some zeros outside the unit circle without putting them somewhere else. We give the name zero guarding to the technique of removing the incorrect zeros of the allpass part of the estimated system.

We have conducted simulations (Björklund, 2002) and tried zero guarding on the Laguerre model structure $($ pole $=0.8$, order $=10)$ with two types of non-minimum phase zeros to remove (ZType): close to only the point +1 or close to the whole unit circle (which includes the point +1 ). We tried seven different distances (ZSize) to be considered as "close". We also tested different maximum number $(Z N o)$ of zeros to remove: 1 to 4 . The closest zeros were removed first. The simulations were conducted in open loop with 1024 trials, three different input signals and four different systems. The SNR was 1.

We utilized the statistical method ANOVA (Analysis of Variance) and confidence intervals (see Montgomery (1997)) to discover statistically significant differences between the different combinations of the levels of the factors ZType, ZSize and ZNo. ANOVA uses the model (here for just two factors):

$$
x_{i j k}=\mu+\tau_{i}+\beta_{j}+(\tau \beta)_{i j}+\epsilon_{i j k},
$$

where $x_{i j k}$ is the response variable (the observation), $\mu$ is the overall mean effect, $\tau_{i}$ is the main effect of the $i^{\text {th }}$ level of the first factor, $\beta_{j}$ is the main effect of the $j^{\text {th }}$ level of the second factor, $(\tau \beta)_{i j}$ is the $i j^{\text {th }}$ interaction effect of the first and second factors and $\epsilon_{i j k}$ are random errors . There are $k$ replicates or number of observations. Hypothesis tests are performed to test all $\tau_{i}=0$, all $\beta_{j}=0$ and all $(\tau \beta)_{i j}=0$ against the opposite cases. With the aid of ANOVA, we can say whether there are statistically significant differences between the levels. In our analysis $x_{i j k}$ was an estimate of the RMS error of the time-delay estimate. The 1024 trials were split into four replicates with each 256 trials which were used to estimate the RMS error. For the factors and interactions with significant differences we computed confidence intervals with a simultaneous confidence level of $95 \%$. If the intervals are non-overlapping there is a significant difference between the levels and we can see which level is the "best". If the intervals are overlapping we cannot say which level, if any, is the best.

The result showed that there were several "good" combinations of ZType, ZSize and ZNo with no significant differences. It was, however, clear that removing zeros close to +1 is better than removing zeros close to the unit circle. It was also clear that removing more than one zero is often necessary, probably because complex valued zeros come in complex conjugated pairs. However, there were no significant differences between removing 2, 3 or 4 zeros but there was a non-significant trend that more zeros are better. If we allow many zeros to be removed, the number of removed zeros will still be limited by the distance ZSize. It is unclear if we can remove too many zeros within the allowed distance. There were significant differences between different distances ZSize. It is not clear what determines the optimal distance. Our advice it that the distance should be optimized by the user for each new application, e.g. with simulations and confidence intervals as in Björklund (2002). It is possible that the optimal distance depends on the speed (the zeros and poles) of the continuous-time system and the sampling interval. This thought is motivated by the relation between the poles $\bar{\lambda}_{i}$ of a continuous-time system and the poles $\lambda_{i}$ of its sampled version: $\lambda_{i}=\exp \left(\bar{\lambda}_{i} T_{s}\right)$ if the two systems are controllable and observable (Glad and Ljung, 2000). Then, for example, the optimal distance should be $k_{1} \exp \left(k_{2} T_{s}\right)$, where $k_{1}$ and $k_{2}$ are some constants.

We chose one of the good combinations of ZType $(+1)$, ZSize (0.15) and ZNo (3) and used it in open loop and 
closed loop simulations with good results (Björklund, 2002, 2003; Björklund and Ljung, 2003). The same ZType, ZSize and ZNo worked for different systems and SNRs and for several model structures and input signals (Björklund, 2003). This value of ZSize, 0.15, is a suggestion to start with.

\section{Discussion}

As said, the method Laguerre DAP (without zero guarding) showed very good results in Horch (2000); Isaksson et al. (2001) for both open loop and closed loop for input and reference signals in the form of steps. They have, however, only tested with step signals and not discovered that the DAP method sometimes fails. In Björklund (2003); Björklund and Ljung (2003), where an extensive evaluation of several time-delay estimation methods was conducted, some results were that DAP methods (with zero guarding) are among the better regarding estimation quality and that the Laguerre model structure is suitable for DAP methods. Another advantage with DAP methods is that they can estimate sub-sample delays, which is necessary in many applications.

If an upper bound exists on the time-delay of the system to investigate, an alternative or complement to the above zero guarding would be to remove those zeros which give a DAP estimate that is less than the bound. The bound can often be chosen high, as the example in Figure 4 shows. A second alternative would be to remove those zeros that makes the DAP estimate closest to the estimate of another time-delay estimation method. Also, if the number of correct zeros in the allpasspart is known, the excess zeros closest to the point +1 could be removed. These three alternatives can still be called "zero guarding". They can also be used to choose the distance ZSize.

\section{Conclusions}

We draw the following conclusions:

- DAP (Discrete-time Allpass part Phase) methods are non-robust and can totally fail in some cases. The reason is the noise moving zeros across the unit circle.

- The failure probability of a DAP method depends on the used model structure, the input signal type and the SNR.

- In failing cases, DAP methods can be made more robust by zero guarding, which means removing incorrect zeros in the estimated allpass system.

- An appropriate choice of zero guarding appears to be robust and work for different systems and SNRs and for several model structures and input signals, in both open loop and closed loop.

- For zero guarding of Laguerre DAP, removing zeros outside the unit circle close to +1 works better than removing zeros outside but close to the whole unit circle. We must also allow more than one zero to be removed. There are significant differences between different distances from the point +1 within which to remove zeros.

- We give the following practical advice on how to use zero guarding of Laguerre DAP: Remove zeros close to +1 . Allow up to 4 zeros to be removed. The distance within which to remove zeros, ZSize, should be optimized in each application. Start with the value 0.15.

- Laguerre DAP with zero guarding seems to be a reliable time-delay estimation method for open loop and closed loop with good estimation quality and the possibility to estimate subsample delays.

\section{Acknowledgements}

This work was supported by The Swedish Research Council (VR).

\section{References}

Björklund, S., Oct. 2002. Analysis of a phase method for time-delay estimation. Tech. Rep. LiTH-ISYR-2467, Dep. EE, Linköping University, Sweden, $<$ www.control.isy.liu.se/publications/doc?id $=1390>$.

Björklund, S., 2003. A survey and comparison of time-delay estimation methods in linear systems. Licentiate thesis LIU-TEKLIC-2003:60, Dep. EE, Linköping University, $<$ www.control.isy.liu.se/publications/doc?id=1599>.

Björklund, S., Ljung, L., December 2003. A review of time-delay estimation techniques. In: Proc. 42nd IEEE Conf. Decision \& Control. Maui, HI, USA, pp. 2502-2507.

CSTB, 4.2.1. Matlab control system toolbox, v. 4.2.1 (R11.1). The Mathworks Inc.

Glad, T., Ljung, L., 2000. Control Theory. Multivariable and Nonlinear Methods. Taylor \& Francis, ISBN 07484-0878-9.

Horch, A., 2000. Condition monitoring of control loops. Phd thesis TRITA-S3-REG-0002, Dep. Signals, Sensors \& Systems, Royal Institute of Technology, Stockholm, Sweden.

Isaksson, A. J., Horch, A., Dumont, G. A., June 2001. Event-triggered deadtime estimation from closed-loop data. In: Proc. American Control Conf. Arlington, VA, USA, pp. 3280-3285.

Ljung, L., 1999. System Identification: Theory for the User, 2nd Edition. Prentice-Hall.

Ljung, L., Glad, T., 1994. Modeling of Dynamic Systems. Information and System Sciences Series. PrenticeHall, Englewood Cliffs, N.J. USA, ISBN 0135970970.

Montgomery, D. C., 1997. Design and Analysis of Experiments. Wiley, ISBN 0-471-15746-5.

Wahlberg, B., May 1991. System identification using Laguerre models. IEEE Transactions on Automatic Control AC-36 (5), 551-562. 


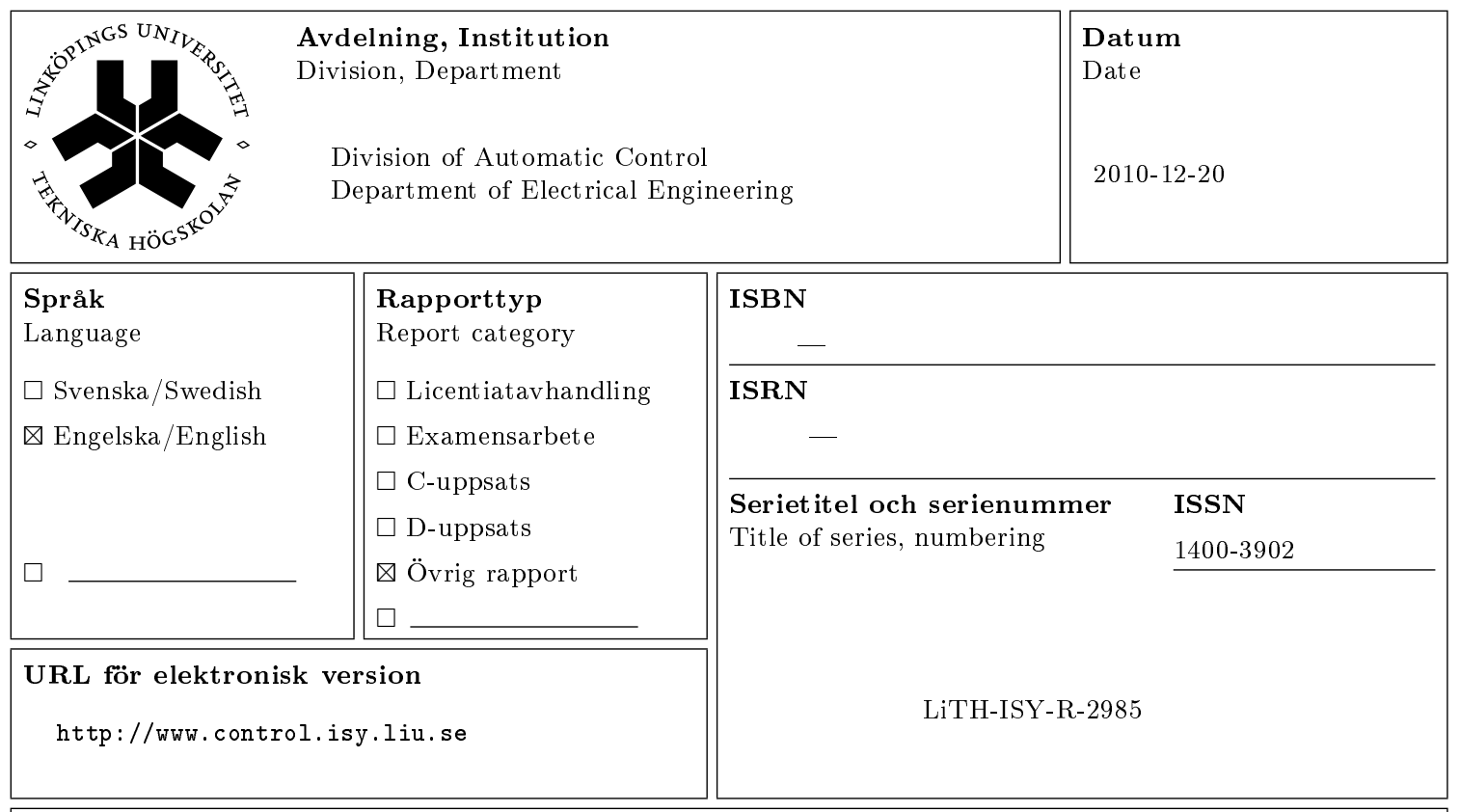

Titel An Improved Phase Method for Time-Delay Estimation

Title

An Impoved Phase Method for Time-Delay Estimation

Författare Svante Björklund, Lennart Ljung

Author

Sammanfattning

Abstract

A promising method for estimation of the time-delay in continuous-time linear dynamical systems uses the phase of the allpass part of a discrete-time model of the system. We have discovered that this method can sometimes fail totally and we suggest a method for avoiding such failures. 